Published in final edited form as:

Int J Med Inform. 2017 May ; 101: 68-74. doi:10.1016/j.ijmedinf.2017.02.005.

\title{
Mobile application for diabetes self-management in China: Do they fit for older adults?
}

\author{
Chenchen Gao ${ }^{a}$, Lanshu Zhou ${ }^{b}$, Zhihui Liuc, Haocen Wang ${ }^{d}$, and Barbara Bowers ${ }^{\mathrm{e}}$ \\ aSchool of Nursing, Second Military Medical University, Shanghai, China \\ bSchool of Nursing, Second Military Medical University, Shanghai, China \\ 'School of Nursing, Second Military Medical University, Shanghai, China \\ dSchool of Nursing, University of Wisconsin-Madison, Wisconsin, American \\ eSchool of Nursing, University of Wisconsin-Madison, Wisconsin, American
}

\section{Abstract}

Objectives-Despite the exponential proliferation of Chinese diabetes applications, none are designed to meet the needs of the largest potential user population. The purpose of this study is to examine the features and contents of Chinese diabetes mobile applications in terms of their suitability for use by older adults with diabetes.

Methods-A search of the Apple application store and the 360 Mobile Assistant was conducted to identify Chinese diabetes applications. Next, we compared the features and contents of all the included and most popular diabetes applications with both the International Diabetes Federation (IDF) clinical guideline and recommended usability criteria for older adults respectively.

Results-Seventy-one diabetes apps were randomly selected (from a pool of 552 diabetes apps) and reviewed. The features of most apps failed to include content areas of known importance for managing diabetes in older adults. Usability of all tested applications was rated moderate to good.

Conclusions-Designing maximally effective medical applications would benefit from attention to both usability and content guidelines targeted for the largest potential user population. Despite the preponderance of older adults in the potential user group, failing to consider the relevance of

\footnotetext{
Corresponding author: Lanshu Zhou, School of Nursing, Second Military Medical University, 800 Xiangyin Rd, Yangpu Dt, Shanghai, China, zhoulanshu@ @otmail.com, Telephone: 86-21-81871511.

Publisher's Disclaimer: This is a PDF file of an unedited manuscript that has been accepted for publication. As a service to our customers we are providing this early version of the manuscript. The manuscript will undergo copyediting, typesetting, and review of the resulting proof before it is published in its final citable form. Please note that during the production process errors may be discovered which could affect the content, and all legal disclaimers that apply to the journal pertain.

Conflicts of interest

None.

Authors' contributions

Conception and design of study: CG, LZ, BB

Acquisition of data: CG, LZ, ZL

Analysis and interpretation of data: CG, LZ, ZL, HW, BB

Drafting article: CG, LZ

Revising article critically for important intellectual content: CG, LZ, ZL, HW, BB

Final approval of version to be submitted: CG, LZ, ZL, HW, BB
} 
content, in addition to usability for the specific population will ultimately limit the usefulness of the app.

\section{Keywords}

Diabetes mellitus; self management; mobile application; older adults; user group

\section{Introduction}

China has the greatest number of people with diabetes in the world (110 million) [1], among whom people over 60 years account for $80.8 \%$ [2]. This number will continue to rise as the population ages [1]. Self-management has proven to be an essential component in diabetes care. While self-management support programs for diabetes such as Happy Life Club ${ }^{\mathrm{TM}}$ [3,4], Patient Empowerment Program [5,6] and Peer Leader-Support Program [7] have shown promising results, they require participants to join in real-time coaching or activities, which challenge older people who are geographically distant, disabled, unable to attend sessions, or prefer not to participate in groups.

Mobile applications (apps) have the potential to provide needed support for older adults with diabetes. Preliminary research results for mobile app use suggest great potential for enhancing the outcomes in diabetes patients [8,9]. There are currently more than 3000 Chinese medical apps in the app store [10], of which diabetes self-management apps comprise a large share. Most of the existing diabetes apps support self-management tasks such as blood glucose testing, physical exercise tracking, insulin and other medication administration, diet, and general diabetes education [11,12]. However, none described in the literature or available for commercial use target the older population.

As both disease management and usability differ for older adults, compared to younger people, designing an effective and useful app for older diabetic patients requires careful attention to both content and usability. Evidence based guidelines for management of diabetes in older adults differ significantly from those for younger diabetics. Older adults are likely to have multiple co-occurring conditions, are likely to experience age related sensory and cognitive changes[13,14], have increased risk of medication side effects and falls [15], and are more likely to experience depression[16-18].

In addition to content tailored for older adult users, there are recommended usability criteria for older adult users $[19,20]$. In terms of features, common age related changes in vision, hearing, cognition, memory, problem solving ability and manual dexterity are relevant for app design [19,21]. Meeting appropriate usability criteria for included features determines patient engagement, optimal benefit of an intervention, and an understanding of generalizability [22]. Isaković's research showed that app features developed for the general population are not necessarily suitable for elderly users [23].

Until now, to our knowledge, only two articles have reported studies of diabetes app usability for the elderly. Isaković et al [23] recruited older adults to evaluate diabetes app they developed for general diabetic patients, reporting that elderly users had difficulty using the app. Arnhold et al [20] performed an expert-led usability test of general diabetes apps for 
patients age 50 years or older; with results showing moderate to good usability. Despite the great number of diabetes apps in China, usability evidence for Chinese older adults is absent.

The field of medical apps is currently one of the most dynamic in app development and medicine [24,25], with real potential to change the way evidence-based healthcare is delivered [24]. Although there is no evidence-based guideline specifically for diabetes apps targeted for older adults [26], there is an existing global clinical guideline for managing older people with diabetes[27] and mobile app usability criteria that includes basic usability for interaction processes, interface design, and comprehensibility of content both in general and specifically for the elderly [20]. Combining usability and clinical content recommendations in developing the app has the potential to improve the uptake and usefulness of apps with also having a positive impact on clinical outcomes.

The purposes of this paper are to (1) provide an overview of features and content of currently available Chinese diabetes self-management apps; (2) examine whether the contents of diabetes apps reflects clinical issues specific to older adults; and (3) evaluate whether popular Chinese diabetes apps fit the usability requirements for older adults.

\section{Method}

We began with an identification of features and contents found in existing Chinese diabetes apps, proceeded to a comparison of evidence-based guidelines for self-managing diabetes in older adults with content in existing diabetes apps, and finally compared app features to usability guidelines and recommendations for older adult populations.

\subsection{Review current commercial diabetes apps}

Data source-Given that iOS and Android are the two most commonly used mobile operating systems, we focused our search on the Apple App Store and 360 Mobile Assistant (the biggest Android platform in China) during September 2015 using keywords "diabetes mellitus" and "blood sugar".

Inclusion and exclusion criteria-A diabetes app was included in our review if (1) it was used for diabetes self-management and included at least one of the following components: blood glucose testing, dietary and physical activity management, medication taking and foot care; (2) intended exclusively for diabetes patients; and (3) ran on mobiles. Apps were excluded if (1) there was no Chinese-language user interface; (2) they were designed for general health management or general chronic disease management; (3) they only provided information, or could only be used for calculation or communication; (4) were exclusively for professional health providers; or (5) could not be opened and run due to technological issues. No apps intended for older users were found.

Selection and extraction-A first random sample selection of 50 apps (from a pool of 552 diabetes apps) in the app store was conducted by two reviewers independently with Joint-Probability of Agreement of 0.9. Descriptions of diabetes apps in the app store were reviewed and filtered based on the inclusion and exclusion criteria. Disagreements on inclusion were resolved by revisiting the inclusion and exclusion criteria, or turning to a 
third reviewer. A second random sample selection of 50 additional apps was conducted with Joint-Probability of Agreement was 1.0. The primary author was responsible for reviewing these apps. Apps were downloaded and installed on reviewers' mobile phone to facilitate analysis. Two reviewers each checked the apps by operating every module, and extracting details including: name, version, operating system, cost and features.

\subsection{Comparison with evidence-based guideline}

The global guideline for managing older people with type 2 diabetes [27] developed by the International Diabetes Federation (IDF) was used to determine content relevance for diabetes self-management.

\subsection{Evaluation of popular diabetes apps}

Based on the rankings in App Annie, the largest app intelligence platform for apps analytics, we selected those ranked in the top 500 medical apps in iOS operating system for usability testing. We employed the usability assessment criteria developed by Arnhold et al [20], that consists of four main criteria (comprehensibility, presentation, usability and general characteristics), 11 subcriteria and 18 items with 5 Likert-scales and dichotomous scales. For example, when we evaluated "Reducing probability of erroneous data input by limiting choice to meaningful values" (under the criterion of "general characteristics" and the subcriterion of "high fault tolerance/efficient fault management"), we checked the range of parameters of every input. For example, we reversed the systolic and diastolic blood pressure entered into apps to identify whether error reports would be generated, examined limitations to entry and explored the availability of automatic entry. If an app met all of the characteristics, we coded it 5 . Throughout the process, two reviewers assessed usability. Each item was independently scaled and average scores were obtained. The inter-rater reliability was measured using intra-class correlation.

\section{Results}

\subsection{Overview of features and contents in current diabetes apps}

Seventy-one apps were reviewed (Figure 1). Among them, 56 were available in iOS, 36 in Android, and 21 in both operating systems. The majority (69/71) were free, and only $2.8 \%$ (2/71) required purchase.

The most common features were data tracking (focusing on self-monitoring of blood glucose, medications, physical activity, diet, and other laboratory indices), patient education, professional consultation, assessment, social support, reminders, data entry automation and data export. In total, $26.8 \%$ (19/71) were limited to one or two features, $39.4 \%(28 / 71)$ provided three or four features, five or six features were offered by $32.4 \%(23 / 71)$ of apps, and only $1.4 \%$ (1/71) offered seven or more features.

\subsubsection{Data tracking}

Self-monitoring of blood glucose: Blood glucose recording was included in almost all apps (70/71). Most apps offered instant feedback for glucose level and advice or encouragement. 
Medications: Medication tracking was included in 62.0\% (44/71) of diabetes apps. Users were encouraged to enter or choose the medication name, dose and frequency, or to upload pictures of medications. Two apps restricted medications to only insulin, and eight could not accommodate recording medications for other diseases.

Physical activity: Exercise records enabling users to track what, when and how long they performed a physical activity was available on 54.9\% (39/71) of apps.

Diet: Fifty percent (36/71) of the apps offered diet management assistance. Of these, 67\% (24/36) allowed users to choose from the food database and record quantities of food, automatically calculating calories. Only $11.1 \%(4 / 36)$ allowed users to enter the name and quantity of food, while $22.2 \%$ (8/36) allowed uploading of pictures and descriptions to share with others.

Laboratory tests: Some apps also included data entry for blood pressure, weight, emotional status, smoking, drinking, HbA1c and other laboratory tests. Continuous data were usually presented graphically, or supplemented with an analysis report.

Data entry automation, data export and reminders: Connectivity to external devices (mostly blood glucose meters, blood pressure monitors, weight scales and sensors) and internal sensors (mostly embedded pedometers) were available on $39.4 \%$ (28/71) and 18.3\% (13/71) of apps respectively. Only $21.1 \%$ (15/71) of apps had data export ability via text message, email, designated website, social network software or mobile SD card. Thirty seven apps included reminders for predetermined self-management behaviors including SMBG, monitoring blood pressure, taking medicines, exercising, conducting a physical exam and other custom task settings.

3.1.2. Education-Diabetes education was found in $56.3 \%$ of apps (40/71), mostly focusing on basic knowledge about diabetes, medication self-management, exercise, diet, self-monitoring blood glucose and diabetic complications. Recipes and peer stories were also included in $19.7 \%$ of apps (14/71). Pictures, cartoons, and videos were used in 4 apps to present information in a non-text format. Information push services regarding new research/ information about daily care for diabetes was employed to complement the stationary knowledge in apps. Some apps delivered push notices based on category, but others were based on the release date.

3.1.3. Professional consultation-Nearly half of the apps (32/71) provided access to professional consultants (mostly physicians, but also dieticians and nurses) who were employed in hospitals or by technology companies. Users could select a professional as their private doctor or consult with the designated professionals using online chat, sending imagetext information, phone calls or emailing data, providing them with tailored advice. Of these, $15.5 \%$ (11/71) required payment for consultation.

3.1.4. Assessment-Assessments were available on $21.1 \%$ (15/71) of apps, generally falling into five categories: risk for diabetes, severity of diabetes, risk for diabetes complications, evaluation of lifestyle, and depression. The contents of assessments included 
laboratory tests, symptoms of diabetes, medical history, lifestyle, and scales such as Mood Disorder Questionnaire (MDQ) and Patient Health Questionnaires (PHQ-9). According to predefined algorithms, assessment reports focused on present health status, advice on examination and screening, diet and exercise, and goal setting, all of which can be created and sent to the user.

3.1.5. Social support-Eighteen percent (13/71) of apps supported communication with families, friends and peers through adding friends, creating diabetes groups for instant communication, or joining a forum to share experiences and feelings. Seven percent (5/71) of apps could warn family members or friends about an abnormal metabolic index via text message, email, or Wechat.

\subsection{Gaps between the diabetes apps and evidence-based guideline}

The global guideline for managing older people with type 2 diabetes covers 22 areas (http:// www.idf.org/sites/default/files/IDF-Guideline-for-older-people-T2D.pdf). Fourteen areas were excluded because they involved clinical treatment recommendations from health care providers $(n=12)$, recommendations for the end of life care $(n=1)$, or recommendations for aged care home $(n=1)$. Evidence-based recommendations in the either areas related to selfmanagement were extracted from the guideline (Table 1).

The contents of the apps only partially addressed the guideline recommendations for older people with diabetes. Specific clinical issues common to older adults with diabetes, such as sexual health, falls and pain were almost completely missing from the diabetes apps. Other important issues such as expression and hypoglycemia were inadequately addressed. For example, only one app asked specifically about depression problems. None of the apps offered hypoglycemia risk assessment. Most apps supported diet recording focusing on total calories rather than nutritional balance and fluid intake, which were largely overlooked. Although most of the apps provided education, content in most apps failed to cover several of the areas identified in the clinical guideline. In addition, while the guideline recommends involving caregivers in daily care and decisions, only a few of the apps included a sharing function, and not all included education for caregivers.

\subsection{Usability test of seven popular diabetes apps}

Within the group of 71 apps, the top seven (based on App Annie ranking) all demonstrated moderate to good (Table 2) usability. The mean average value of comprehensibility, presentation, usability and general characteristics were 3.9, 3.7, 3.4 and 3.0 out of 5, respectively. The subcriteria "sufficient color contrast", "use of understandable semantics" and "password-protected services" were scored higher with an average value of 4.3, 4.1 and $85.7 \%$, respectively. The subcriteria "high fault tolerance/efficient fault management", "large size of operating elements", "instant and easily understandable feedback" and "ability to adapt the size of operating elements and displayed images" were scored lower, with an average value of 3.0, 3.1, 3.1 and 42.9\%, respectively. Among the seven apps, Zhang shang tang yi received the highest average usability score of 3.8, while Da yi sheng received the lowest score of 3.4. Inner-rated reliability ranged from 0.806 to 0.916 . 


\section{Discussion}

This study revealed that most Chinese diabetes apps are multi-featured, but that the features related to specific clinical issues common to older adults with diabetes were mostly absent from existing diabetes apps. Most were inadequate for both older adults and their caregivers. Thus, commercial diabetes apps designed for general diabetic patients frequently lack specificity for clinical issues relevant to older adults.

The contents of apps influence the quality and safety issue for older adults with diabetes to use, and also determine the intervention outcome. There is evidence that many apps developers have little or no formal medical training, do not involve clinicians in the development process and may therefore be unaware of patient safety/population specific clinical issues [28-30]. Absence of these functions prevents older adults from being informed about potential risks or gravity of symptoms, putting them at risk for negative health outcomes [31]. Depression shares many symptoms with diabetes, such as fatigue, weight loss and sleep disturbance [27], and is strongly associated with increased burden of diabetes symptoms [32], incurring increased risk of diabetes complications [33]. However, assessments of depression are generally lacking in diabetes apps. Providing a periodic assessment of risk may enable older adults or their caregivers to set up early-warning awareness of signs and symptoms, speeding contact with their healthcare professionals, reducing time to adjustments in their treatment plans. Older adults are often unwilling to inform healthcare providers about their health condition during regular visits (often denying pain [27] and reluctant to discuss sexual problems [34]). Including self-assessment and feedback in apps could provide a comfortable way for older adults to identify health conditions without requiring face-to-face interactions with healthcare providers.

Compared with past literature reviews [11,12,35], Chinese diabetes apps are more likely than foreign language diabetes apps to contain education, but are similar in their lack of individualized education and topics specific to older adults. We suggest that future diabetes

apps for older adults could add a transfer link to tailored or individualized contents based on assessments and daily data recording. Educational contents that specific to older adults resulting from diabetes, such as sexual health, falls and pain need to be included. The information should be easy to read and be categorized by theme. Researchers need also find out the way that older adults would identify the themes in apps. Another key recommendation involves education for informal caregivers of older adults (the primary source of everyday advice, emotional support, and practical help for older adults [27]). Considering memory decline in older people, it is advisable to periodically send reviews and summaries of key points and to send relevant information to caregivers.

The moderate to good outcome for the usability evaluation was similar to findings reported by Arnhold et al [20] and by Demidovich et al [35]. Our results indicated that the size of operating elements, fault tolerance, and instant feedback of diabetes apps need to be strengthened. Firstly, the font size and screen in some apps were not large enough, creating an obstacle for older adults with vision impairment or decreased manual dexterity, and may increase the possibility of erroneous input. This is a major problem experienced by older adults using mobile technology [36]. Ability to adapt the size of operating elements and 
displayed images could help to eliminate the restriction created by limited screen size. This feature is generally used in the education module. Hence, increasing the size of operating elements is a priority for improving usability. In addition, adding reading feature to apps is an optimal alternative for older adults with visual problems and low literacy.

Secondly, fault management restricting choices for data entry and warning reports were not present in some apps, allowing faulty data input, which can be dangerous when used for decision support. Applying automatic data entry is recommended in order to decrease the possibility of faulty data, and to simplify the cumbersome process of logging data. It is also advisable to use voice logging instead of manual entry, and a voice speaker to audit the correction. Additionally, it could be helpful to limit the range of data and offer instant fault feedback when the apps only support manual entry. Lastly, diabetes apps could be used as electronic diaries, providing instant and easily understandable feedback triggered when users input the data.

Findings from this study suggest that many diabetes apps are multi-featured, differing from Arnhold et al's [20] finding that most have only one or two functions. Arnhold et al [20] also found a negative correlation between the number of functions and usability scores. However, this should be interpreted with caution, as usefulness may rely on an even larger set of features. One possibility for addressing this dilemma could be to have greater flexibility and customization. Features not needed by a particular user or at a particular time could be hidden, and reestablished when needed. In order to help older adults with diabetes use multifeatured apps efficiently, providing training and practice before using, improving selfefficacy and attitudes about using technology [37] at the same time.

The methodology used in this study suggests that bringing clinicians, researchers and app developers together. Specifically, taking both the contents and features of diabetes apps into consideration, will maximize diabetes apps quality and usability. This study suggests a process that can be used in the development of medical apps. However, the study has several limitations. First, apps developed or updated after October 2015 were not included. Second, even though the usability evaluation was based on prior recommendations for usability in older populations, there is currently insufficient research to develop a best practice guideline for usability with this population.

\section{Conclusion}

Medical apps development is a rapidly growing field in China, with great potential to proliferate. The currently available Chinese diabetes apps are not well designed for older adults, the largest potential user group. Specifically, the content and usability specific to this audience have not been consistently integrated into apps designs. One feasible way to determine content is to identify population specific national or international clinical guidelines prior to app development[12,38,39]. Unfortunately, currently accepted clinical guidelines do not appear to have been consulted in the design of most diabetes apps. Research is also available on usability for older adult (as well as other) populations. Uptake and continued use of medical apps could have a profound impact on health, but will only be achieved by attending to evidence based standards on both usability and content. 


\section{Acknowledgments}

This project was supported by the Shanghai Health System Outstanding Academic Leader Training Program [grant XBR2013108]; and the Shanghai Academic/Technical Research Leader Program [grant 16XD1403200]; and the Clinical and Translational Science Award (CTSA) program, through the NIH National Center for Advancing Translational Sciences (NCATS) [grant UL1TR000427].

\section{References}

1. International Diabetes Federation: IDF diabetes atlas- $7^{\text {th }}$ edition. http://www.diabetesatlas.org/ (2015, accessed 8 Mar 2016)

2. Chan JC, Zhang Y, Ning G. Diabetes in China: a societal solution for a personal challenge. The lancet Diabetes \& endocrinology. 2014; 2(12):969-979. [PubMed: 25218728]

3. Browning CJ, Yang H, Zhang T, et al. Implementing a chronic disease self-management program into china the happy life club ${ }^{\mathrm{TM}}$. Front Public Health. 2015; 27(2):181.

4. Browning C, Chapman A, Yang H, et al. Management of type 2 diabetes in China: the Happy Life Club, a pragmatic cluster randomised controlled trial using health coaches. BMJ open. 2016; 6(3):e009319.

5. Wong CKH, Wong WCW, Lam CLK, et al. Effects of Patient Empowerment Programme (PEP) on Clinical Outcomes and Health Service Utilization in Type 2 Diabetes Mellitus in Primary Care: An Observational Matched Cohort Study. M.E. Khamseh. PLoS ONE. 2014; 9(5):e95328. [PubMed: 24788804]

6. Wong CKH, Wong WCW, Wan YF, et al. Patient Empowerment Programme in primary care reduced all-cause mortality and cardiovascular diseases in patients with type 2 diabetes mellitus: a population-based propensity-matched cohort study. Diabetes, Obesity and Metabolism. 2015; 17(2): 128-135.

7. Zhong X, Wang Z, Fisher EB, et al. Peer Support for Diabetes Management in Primary Care and Community Settings in Anhui Province, China. The Annals of Family Medicine. 2015; 13(Suppl_1):S50-S58. [PubMed: 26304972]

8. Holtz B, Lauckner C. Diabetes Management via Mobile Phones: A Systematic Review. Telemedicine and e-Health. 2012; 18(3):175-184. [PubMed: 22356525]

9. Garabedian LF, Ross-Degnan D, Wharam JF. Mobile Phone and Smartphone Technologies for Diabetes Care and Self-Management. Current Diabetes Reports. 2015; 15(12)

10. National Health and Family Planning Commission of the People's Republic of China:“互联网 +” 使求医问药更便捷. http://www.nhfpc.gov.cn/zhuzhan/mtbd/ 201604/9a98237b2e4842349ed1b8b78bd22016.shtml. (2016, accessed 8 Dec 2016)

11. El-Gayar O, Timsina P, Nawar N, et al. Mobile applications for diabetes self-management status and potential. J Diabetes Sci Technol. 2013; 7(1):247-262. [PubMed: 23439183]

12. Chomutare T, Fernandez-Luque L, Arsand E, et al. Features of mobile diabetes applications: review of the literature and analysis of current applications compared against evidence-based guidelines. Journal of medical Internet research. 2011; 13(3):e65. [PubMed: 21979293]

13. Kirkman MS, Briscoe VJ, Clark N, et al. Diabetes in Older Adults. Diabetes Care. 2012; 35(12): 2650-2664. [PubMed: 23100048]

14. Weinger K, Beverly EA, Smaldone A. Diabetes Self-Care and the Older Adult. Western Journal of Nursing Research. 2014; 36(9):1272-1298. [PubMed: 24510969]

15. Huang ES, Karter AJ, Danielson KK, et al. The association between the number of prescription medications and incident falls in a multi-ethnic population of adult type- 2 diabetes patients: the diabetes and aging study. Journal of General Internal Medicine. 2010; 25(2):141-146. [PubMed: 19967465]

16. Bruce DG, Casey GP, Grange V, et al. Cognitive impairment, physical disability and depressive symptoms in older diabetic patients: the Fremantle Cognition in Diabetes Study. Diabetes Research and Clinical Practice. 2003; 61(1):59-67. [PubMed: 12849924] 
17. Bell RA, Smith SL, Arcury TA, et al. Prevalence and correlates of depressive symptoms among rural older African American native Americans and whites with diabetes. Diabetes Care. 2005; 28(4):823-829. [PubMed: 15793180]

18. Chou K-L, Chi I. Prevalence of depression among elderly Chinese with diabetes. International Journal of Geriatric Psychiatry. 2005; 20(6):570-575. [PubMed: 15920708]

19. Zaphiris P, Ghiawadwala M, Mughal S. Age-centered research-based web design guidelines. CHI'05 extended abstracts on Human factors in computing systems. ACM. 2005:1897-1900.

20. Arnhold M, Quade M, Kirch W. Mobile applications for diabetics: a systematic review and expertbased usability evaluation considering the special requirements of diabetes patients age 50 years or older. Journal of Medical Internet Research. 2014; 16(4):e104. [PubMed: 24718852]

21. Sonderegger A, Schmutz S, Sauer J. The influence of age in usability testing. Applied Ergonomics. 2016; 52:291-300. [PubMed: 26360221]

22. Lyles CR, Sarkar U, Osborn CY. Getting a Technology-Based Diabetes Intervention Ready for Prime Time: a Review of Usability Testing Studies. Current Diabetes Reports. 2014; 14(10)

23. Isaković M, Sedlar U, Volk M, et al. Usability Pitfalls of Diabetes mHealth Apps for the Elderly. Journal of Diabetes Research. 2016; 2016:1-9.

24. Buijink AWG, Visser BJ, Marshall L. Medical apps for smartphones: lack of evidence undermines quality and safety. Evidence Based Medicine. 2013; 18(3):90-92. [PubMed: 22923708]

25. Lewis TL, Wyatt JC. mHealth and Mobile Medical Apps: A Framework to Assess Risk and Promote Safer Use. Journal of Medical Internet Research. 2014; 16(9):e210. [PubMed: 25223398]

26. Murfin M. Know your apps: an evidence-based approach to evaluation of mobile clinical applications. The Journal of Physician Assistant Education. 2013; 24(3):38-40.

27. Sinclair, A., Dunning, T., Colagiuri, S., et al. Managing older people with type 2 diabetes: global guideline. http://www.idf.org/sites/default/files/IDF-Guideline-for-older-people-T2D.pdf. (2013, accessed 8 August 2016)

28. Hamilton AD, Brady RRW. Medical professional involvement in smartphone 'apps' in dermatology: Correspondence. British Journal of Dermatology. 2012; 167(1):220-221. [PubMed: 22283748]

29. Huckvale K, Car M, Morrison C, et al. Apps for asthma self-management: a systematic assessment of content and tools. BMC medicine. 2012; 10(1):1. [PubMed: 22216957]

30. Rodrigues MA, Visvanathan A, Murchison JT, et al. Radiology smartphone applications; current provision and cautions. Insights into Imaging. 2013; 4(5):555-562. [PubMed: 23912880]

31. Sircar M, Bhatia A, Munshi M. Review of Hypoglycemia in the Older Adult: Clinical Implications and Management. Canadian Journal of Diabetes. 2016; 40(1):66-72. [PubMed: 26752195]

32. Ludman EJ, Katon W, Russo J, et al. Depression and diabetes symptom burden. General Hospital Psychiatry. 2004; 26(6):430-436. [PubMed: 15567208]

33. Johnston SS, Conner C, Aagren M, et al. Association between hypoglycaemic events and fallrelated fractures in Medicare-covered patients with type 2 diabetes. Diabetes, Obesity and Metabolism. 2012; 14(7):634-643.

34. Lindau ST, Tang H, Gomero A, et al. Sexuality among middle-aged and older adults with diagnosed and undiagnosed diabetes: a national, population-based study. Diabetes Care. 2010; 33(10):2202-2210. [PubMed: 20802158]

35. Demidowich AP, Lu K, Tamler R, et al. An evaluation of diabetes self-management applications for Android smartphones. Journal of Telemedicine and Telecare. 2012; 18(4):235-238. [PubMed: 22604278]

36. Kurniawan S. Mobile phone design for older persons. Interactions. 2007; 24(4):24-25.

37. Laganà L. Enhancing the Attitudes and Self-Efficacy of Older Adults Toward Computers and the Internet: Results of a Pilot Study. Educational Gerontology. 2008; 34(9):831-843. [PubMed: 20148185]

38. Nguyen AD, Baysari MT, Kannangara DRW, et al. Mobile applications to enhance selfmanagement of gout. International Journal of Medical Informatics. 2016; 94:67-74. [PubMed: 27573313] 
39. Breland JY, Yeh VM, Yu J. Adherence to evidence-based guidelines among diabetes selfmanagement apps. Translational Behavioral Medicine. 2013; 3(3):277-286. [PubMed: 24073179] 


\section{Summary points}

\section{What was already known?}

- $\quad$ Medical apps will be maximally effective and useful if they are informed by evidence relevant to both content and usability.

- The potential user population for self-management of diabetes is increasing significantly.

\section{What this study has added?}

- Demonstration of process that can be used to maximize user group by combining evidence for content and usability.

- Education based on targeted assessments and data recording, can be used to maximize effectiveness of medical apps for patients and their caregivers.

- To increase the flexibility and usefulness of diabetes apps, functions not needed by a particular user or at a particular time could be hidden, and reestablished when needed.

- Usability domains such as size of operating elements, fault tolerance, and instant feedback need to be addressed. 


\section{Highlights}

- Demonstration of process that can be used to maximize user group by combining evidence for content and usability.

- Education based on targeted assessments and data recording can be used to maximize effectiveness of medical apps for older adults and their caregivers.

- Usability domains such as size of operating elements, fault tolerance, and instant feedback need to be addressed. 
Apps Store/Market

Apple Store $=406$

360 Mobile Assistant $=146$

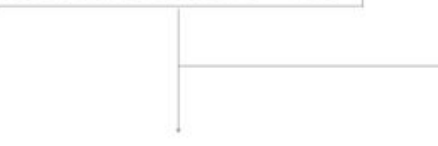

Apps review $=493$

Apps removed $=\mathbf{2 5 6}$

Education and information $=43$

Diet recipe $=21$

General health management or

chronic disease management $=63$

No Chinese $=70$

For health providers $=28$

Can not access $=16$

Diabetes calculation $=14$

Diabetes forum $=1$
Duplicate removed

Apps $=59$

Apps removed $=166$

Irrelevant to diabetes self-

management $=166$

Figure 1.

Diabetes apps selection process. 
Table 1

Comparison of features and contents in diabetes apps with guideline recommendations.

\begin{tabular}{|c|c|c|}
\hline Areas & Guideline Recommendations & $\begin{array}{l}\text { Description of relevant features/contents } \\
\text { in } 71 \text { diabetes apps }\end{array}$ \\
\hline Medication & $\begin{array}{l}\text { - } \\
\text { - } \\
\text { Document the medicine list in a legible form (preferable not } \\
\text { hand written) } \\
\text { Medicine administration time must coincide with meal times } \\
\text { to reduce the risk of hypoglycemia }\end{array}$ & $\begin{array}{l}\text { - } \quad \text { Medication recording : } 44 \\
\text { apps }(62.0 \%) \\
\text { Medication Reminders: } 32 \\
\text { apps }(45.1 \%)\end{array}$ \\
\hline Hypoglycemia & $\begin{array}{l}\text { - } \\
\text { Assess hypoglycemia risk } \\
\text { Every person who is self-administering insulin should have } \\
\text { an evaluation of their self-administration abilities } \\
\text { Caregivers and family should receive education about the } \\
\text { recognition and treatment of hypoglycemia }\end{array}$ & $\begin{array}{l}\text { Providing education about } \\
\text { hypoglycemia appeared in } \\
\text { education }\end{array}$ \\
\hline Nutrition & $\begin{array}{l}\text { - The meal plan should include a variety of foods to ensure } \\
\text { essential vitamins, minerals, protein, and fiber are consumed } \\
\text { in adequate amounts } \\
\text { - A consistent amount of carbohydrate should be provided at } \\
\text { each meal } \\
\text { - } \quad \text { Achieve and maintain a healthy body weight } \\
\text { - } \quad \begin{array}{l}\text { Encourage consumption of adequate amounts of fluid to } \\
\text { avoid dehydration, especially in hot weather }\end{array}\end{array}$ & $\begin{array}{l}\text { Diet recording: } 36 \text { apps } \\
(50.7 \%) \\
\text { - Weight recording: } 30 \text { apps } \\
(42.3 \%) \\
\text { - } \quad \begin{array}{l}\text { Fluid recording: } 1 \text { app } \\
(1.4 \%)\end{array}\end{array}$ \\
\hline Depression & $\begin{array}{l}\text { - } \quad \text { Screening for and monitoring of depressive symptoms } \\
\text { Involvement of the family in recognizing early warning } \\
\text { symptoms and signs of depression }\end{array}$ & $\begin{array}{l}\text { - } \\
\text { Emotion recording: } 11 \text { apps } \\
(15.5 \%) \\
\text { Depression assessment: } 1 \\
\text { app }(1.4 \%)\end{array}$ \\
\hline Pain & $\begin{array}{l}\text { - } \\
\text { Assess pain risk } \\
\text { Provide an appropriate pain assessment tool for people at } \\
\text { moderate to high risk of pain Provide advice on the } \\
\text { suitability of driving while on pain management medicines }\end{array}$ & $\begin{array}{l}\text { Providing education about } \\
\text { pain seldom appeared in } \\
\text { education }\end{array}$ \\
\hline Falls & $\begin{array}{l}\text { Provide education about fall prevention to all older people } \\
\text { with } \\
\text { Provide a referral or intervention to enhance endurance, gait, } \\
\text { balance, and strength training } \\
\text { Undertake home safety checks and modifications in } \\
\text { collaboration with the person with diabetes and their family }\end{array}$ & $\begin{array}{l}\text { Providing education about } \\
\text { falls seldom appeared in } \\
\text { education }\end{array}$ \\
\hline Sexual health & $\begin{array}{l}\text { - Assess sexual health and well-being } \\
\text { - If a sexual health problem is identified, provide appropriate } \\
\text { counseling and management }\end{array}$ & $\begin{array}{l}\text { Providing education about } \\
\text { sexual seldom appeared in } \\
\text { education }\end{array}$ \\
\hline $\begin{array}{l}\text { Education and } \\
\text { self-monitoring } \\
\text { of blood glucose }\end{array}$ & $\begin{array}{l}\text { - } \quad \text { Provide on-going education and review } \\
\text { - } \quad \text { Provide an individualized blood glucose monitoring plan }\end{array}$ & $\begin{array}{l}\text { Providing education: } 40 \text { apps } \\
(56.3 \%)\end{array}$ \\
\hline
\end{tabular}


ᄅ

\begin{tabular}{|c|c|c|c|c|c|c|c|c|c|c|c|c|c|c|c|c|c|c|c|}
\hline & ט & $m$ & $\stackrel{n}{+}$ & $\stackrel{n}{i}$ & $\ddot{n}$ & $\because$ & ナ & $\dddot{n}$ & in & $\ddot{n}$ & z & $n$ & $n$ & + & $\stackrel{n}{n}$ & + & $N$ & $\because$ & $\stackrel{\mathscr{D}}{\Omega}$ \\
\hline & 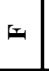 & $m$ & in & 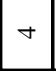 & ナ & $\ddot{m}$ & 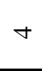 & t & in & $\ddot{m}$ & $\stackrel{\circ}{z}$ & $\ddot{n}$ & $m$ & $\nabla$ & $\ddot{n}$ & $\nabla$ & 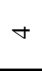 & $i$ & 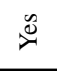 \\
\hline & 디 & $m$ & in & $\nabla$ & rn & $m$ & nq & $\ddot{n}$ & in & $\stackrel{n}{i}$ & $\stackrel{2}{z}$ & $m$ & $m$ & t & $n$ & $m$ & 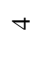 & $\ddot{m}$ & $\triangleq$ \\
\hline 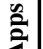 & 0 & $\nabla$ & in & $\stackrel{n}{m}$ & ng & 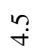 & $\stackrel{n}{q}$ & 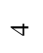 & in & $\ddot{m}$ & స्र & 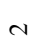 & $\begin{array}{ll}n \\
m \\
n\end{array}$ & $\stackrel{n}{q}$ & 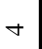 & $m$ & $n$ & - & $\stackrel{2}{z}$ \\
\hline
\end{tabular}

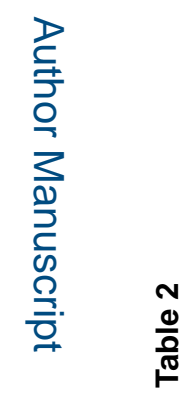

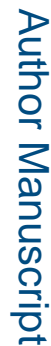

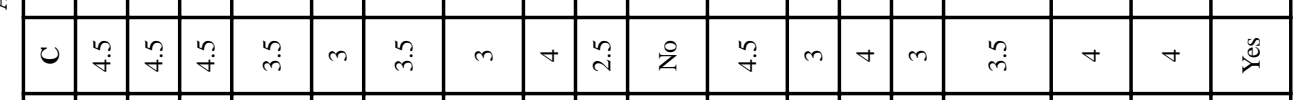

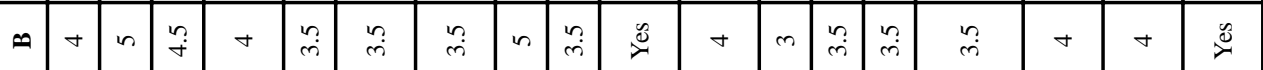

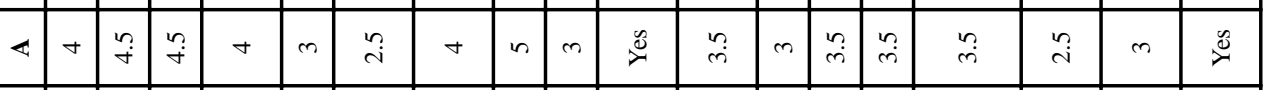

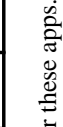

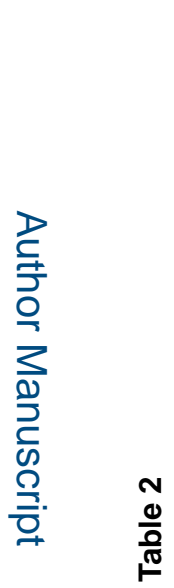

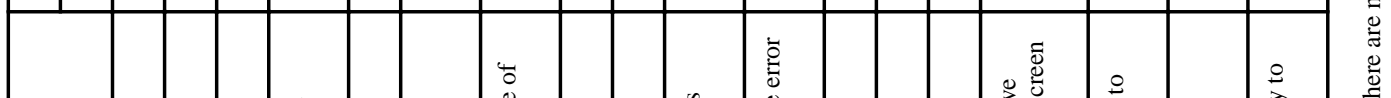

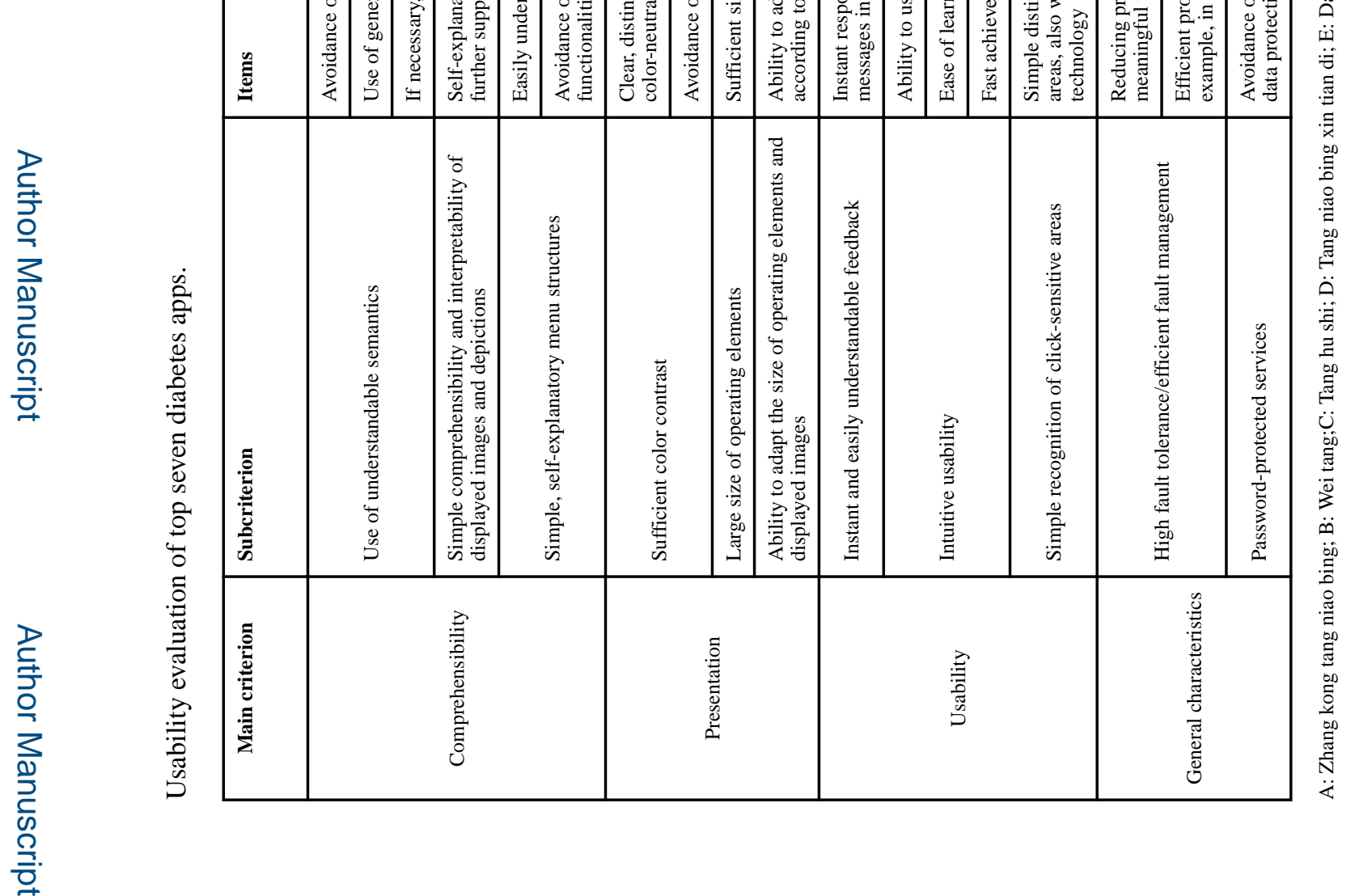

Int J Med Inform. Author manuscript; available in PMC 2018 May 01. 